\title{
Significance of Neuropilin-1 Expression, Ceruloplasmin oxidase and Copper in Egyptian Children with B-Lineage Acute Lymphoblastic Leukemia
}

Nawal M. Abdel Qawy ${ }^{1}$, Alshaymaa M. Alhabibi ${ }^{1}$, Sahar A. Khaliil' ${ }^{2}$ Marwa M. Abdelfattah*3

${ }^{1}$ Department of Clinical Pathology, Faculty of Medicine for Girls, Al-Azhar University,

${ }^{2}$ Department of Pediatric Oncology, National Cancer Institute,

${ }^{3}$ Central Public Health Laboratories, Ministry of Health, Cairo, Egypt

*Corresponding author: Marwa M. Abdelfattah, Mobile: (+20)01003832597, E-mail: mmerom@ hotmail.com

\begin{abstract}
Background: Neuropilins are transmembrane glycoproteins that act as receptors for vascular endothelial growth factors (VEGF) and are involved in the process of tumor angiogenesis. Ceruloplasmin is a member of the multi copper oxidase family. It has antioxidant properties that play a central role in protection of the body against advanced oxidation protein products.
\end{abstract}

Objective: This study was aimed to assess the expression of Neuropilin-1(NRP-1) on blasts of B-lineage precursor lymphoblastic leukemia (ALL) to be used in the diagnostic panel of this disease. We also aimed to assess the alteration of the levels of ceruloplasmin oxidase and copper as a compensatory mechanism to minimize the effects of reactive oxygen species resulting from leukemias.

Subjects and Methods: This study was conducted on 40 children with newly diagnosed B-lineage precursor lymphoblastic leukemia. 40 age-matched controls were enrolled to serve as control. The expression of NRP-1 on peripheral blood samples was evaluated by flow cytometry as the proportion of positive cells expressing the marker. Ceruloplasmin oxidase and copper levels were assessed by immunoturbidimetric assay.

Results: There was highly significant increase in the proportion of positivity of NRP -1 in patients compared with control group $(\mathrm{P}<0.001)$ Ceruloplasmin oxidase and copper levels were also higher in patients compared with control group $(\mathrm{P}<0.001)$.

Conclusions: It could be concluded that NRP-1 is a valuable marker for diagnosis of B-lineage ALL. There is an increase in the levels of ceruloplasmin oxidase and copper which at the time of diagnosis of B-ALL.

Keywords: Neuropilin-1 (NRP-1), Ceruloplasmin oxidase, Copper, B- lineage precursor lymphoblastic leukemia.

\section{INTRODUCTION}

Neuropilins (NRPs) are multifunctional, nontyrosine kinase surface glycoproteins that are expressed in all vertebrates with an important role in a wide range of physiological processes including development, axonal guidance, angiogenesis, immunity, as well as in pathological conditions such as cancer ${ }^{(1)}$.

There are two isoforms, neuropilin-1 (NRP-1 or CD304) and neuropilin-2 (NRP-2). NRP-1 and NRP-2 are expressed in various immune cells, such as macrophages, dendritic cells (DCs), T cells, B cells, and mast cells where they regulate a myriad of functions, including development, migration and recruitment, communication between different immune cells as well as regulation of immune response, under normal physiological condition and during pathological disorders. They are also detected in osteoclasts and osteoblasts where they regulate bone homeostasis ${ }^{(2)}$.

NRP-1 is characterized mainly in T cell subsets, and to a lesser extent in macrophages and DCs. In comparison, NRP-2 is less studied and poorly characterized $^{(3)}$.

NRPs were demonstrated to act as coreceptors for vascular endothelial growth factor (VEGF) and the class III Semaphorin family of molecules by interacting with VEGF receptors and Plexins, respectively. However, other ligands for NRPs have also been reported (4).

VEGF is an important cytokine that contributes to disease evolution in various neoplasms and described as a mediator of leukemia associated angiogenesis as well as asan autocrine growth regulator in leukemic cells. Angiogenesis is an important requirement for the development and progression of hematological malignancies ${ }^{(5)}$.

They are often upregulated in various clinical disorders, including cancer, where they increase the oncogenic activities of malignant cells by promoting survival, inducing angiogenesis and lymphangiogenesis and contribute to therapy resistance ${ }^{(6)}$.

Malnutrition has long been recognized as an important component of adverse outcomes among patients with cancer, including increased morbidity and mortality and decreased quality of life. Different studies tried to establish relationship between trace elements and malignant diseases ${ }^{(7)}$.

Ceruloplasmin is copper binding glycoprotein, it hasantioxidant properties due to its ability to react with and scavenge toxic oxygen species such as superoxide and hydrogen peroxide. An imbalanced production of reactive oxygen species plays a role in the pathogenesis of a number of human diseases as cancer, atherosclerosis, neurodegenerative disease and allergy ${ }^{(8)}$.

The aim of the current study was to study the expression of NRP-1 in patients with B-lineage precursor lymphoblastic leukemia to determine if it could be used for routine diagnosis. Also, it determines the changes in the levels of ceruloplasmin oxidase and copper in those 
patients to assess whether they have any modifying effects regarding the etiology of cancer.

\section{SUBJECTS AND METHODS}

This case-control study included a total of forty patients with B-ALL, attending at Oncology Unit, Pediatric Department, National Cancer Institute. Written informed consent from all the children's guardians were obtained. This study was conducted between June 2016 and March 2019.

Forty samples were collected from age- and gendermatched apparently healthy individuals, who served as controls.

\section{Ethical consideration:}

The study was conducted according to the guidelines of the World Medical Association Helsinki declaration for studies on human subjects.

It was approved by the Institutional Review Board (IRB) of the Clinical Pathology Department of Al-Zahraa University Hospital.

\section{Inclusion criteria:}

Newly diagnosed B-ALL patients (aged up to 12 years) were selected according to 2016 World Health Organization (WHO) criteria (lymphoblasts $\geq 20 \%$ of all nucleated cells).

\section{Exclusion criteria:}

B-ALL Patients receiving treatment or Philadelphia chromosome-positive patients were excluded from this study.

\section{Procedures and variable assessments:}

All studied individuals were subjected to a comprehensive history-taking, detailed clinical examination and the following laboratory investigations.

I. A complete blood count (CBC) was performed with the automated hematology analyzer Sysmex KX 21N (Japan), and biochemical analyses were performed with the Cobas c311 system (Germany) of kidney function parameters, liver function parameters and lactate dehydrogenase (LDH) levels.

II. Bone marrow aspiration (BMA), immunophenotyping using a panel of acute leukemia to confirm the diagnosis (including CD45, CD34, HLA-DR, cytoplasmic TDT, CD117, CD19, CD22, cytoplasmic CD22,CD79a, CD10, CD5, CD3, CD13 and CD33) and genetic studies.

III. Immunophenotyping of NRP-1 (CD304): Immunophenotyping was performed on ethylenediamine-tetra-acetic acid (EDTA) peripheral blood specimens obtained before the administration of any treatment. The samples were analyzed within 24 hours of collection.

Flow cytometry was conducted using four colors FACS Calibur (BD, Biosciences, San Jose, USA). Cell Quest Pro software (BD Biosciences, San Jose, USA) was used for data analysis. Compensation setting was established before acquiring the samples using color calibrite beads (BD, Biosciences, San Jose, USA, lot no. 5093879). After adjusting the sample count for acquisition, unstained samples were acquired to detect the sample auto-florescence. Isotype controls, mouse IgG1 FITC /IgG2a PE control (cat. no. 342409) and mouse IgG APC control (cat. no. 550931) were obtained from BD, Biosciences, San Jose for detection of nonspecific binding.

Then samples were analyzed for the expression of neuropilin-1 by PE conjugated $\mathrm{Ab}$ (R\&D systems, Minneapolis, USA, Cat. no. FAB3870P-025, lot no. AAUA0314101). The cutoff value of positivity of Neuropilin-1 was $20 \%$. The optimal concentration for each antibody dye used was detected by titration experiments in all diagnostic and research markers.

Gating strategy: Using forward and side scatter (FS/SS); then within the CD45/ SSC, initial gating was performed on blast area in cases or lymphocytes in control group in the dot plot graph. Then subset of cells expressing NRP-1 was determined using their percentage (Fig. 1). (A) 

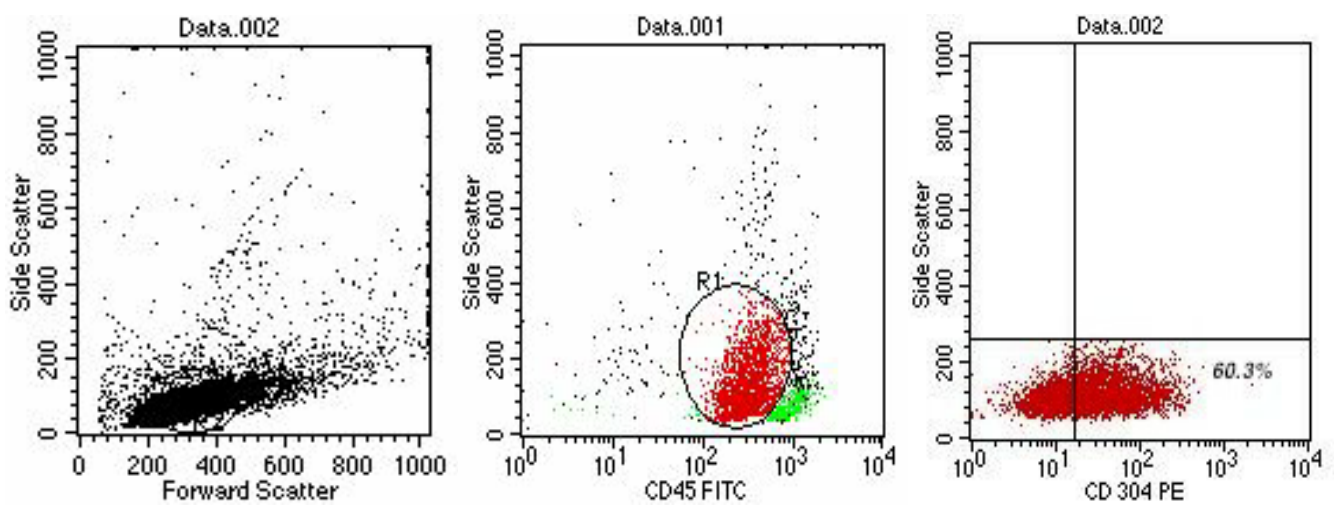

(B)
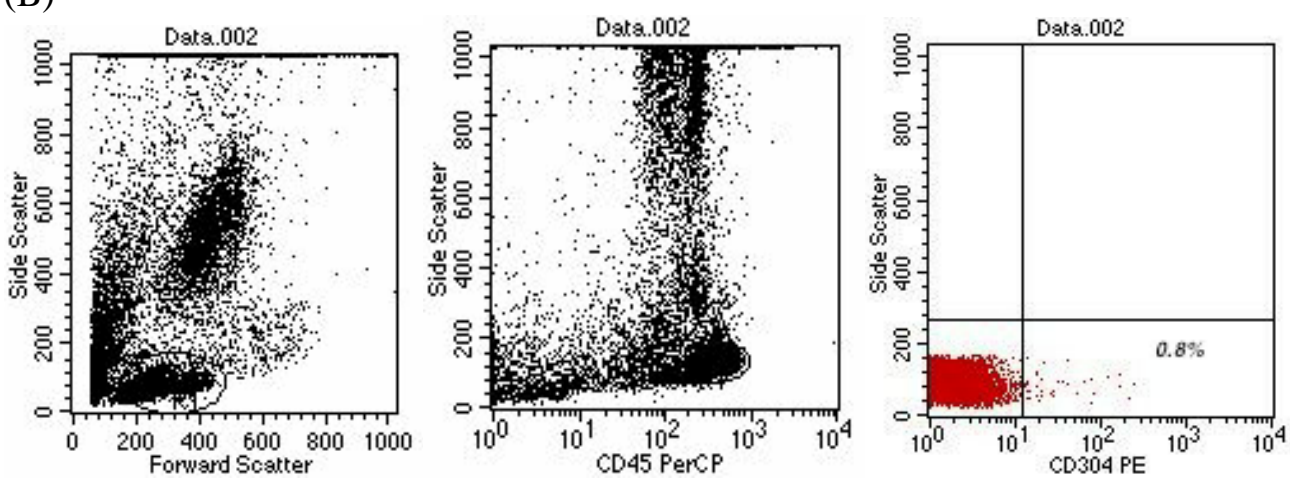

Figure (1): (A) Representative dot blot showing a case of ALL with positive Neuropilin-1 (NRP-1), B) Representative dot blot showing negative control.

IV. Ceruloplasmin oxidase and serum copper were performed using Quantitative determination by using an immunoturbidimetric assay performed by Beckman Coulter AU 680, California, USA.

\section{Statistical methods:}

Data were coded and entered using the statistical package SPSS (Statistical Package for the Social Sciences) version 25. Data was summarized using mean, standard deviation, median, minimum and maximum in quantitative data and using frequency (count) and relative frequency (percentage) for categorical data. Comparisons between groups were done using unpaired $t$ test in normally distributed quantitative variables while non-parametric Mann-Whitney test was used for nonnormally distributed quantitative variables. For comparing categorical data, Chi square (c2) test was performed. Exact test was used instead when the expected frequency is less than 5. Correlations between quantitative variables were done using Spearman correlation coefficient. ROC curve was constructed with area under curve analysis performed to detect best cutoff value of different parameters for detection of cases. Pvalues less than 0.05 were considered as statistically significant

\section{RESULTS}

The study was conducted on 40 newly diagnosed B-ALL patients, including 24 males and 16 females with age range of 2 to 11 years, with a mean age of $7.2 \pm 2.3$ years and a median age of 9 years. 40 samples were used as controls with an age range of 2 to 12 years, a mean age of $6.4 \pm 2.8$ years and a median age of 7 years. Clinical characteristics of the cases and control group are illustrated in Table (1).

Table (1): Clinical characteristics of cases and control group.

\begin{tabular}{|c|c|c|c|}
\hline & & \multicolumn{2}{|c|}{ Case $(n=40)$} \\
\hline & & Count & $\%$ \\
\hline \multirow[t]{2}{*}{ Sex } & Females & 16 & 40 \\
\hline & Males & 24 & 60 \\
\hline \multirow[t]{2}{*}{ Hepatomegaly } & positive & 34 & 85.0 \\
\hline & negative & 6 & 15.0 \\
\hline \multirow[t]{2}{*}{ Splenomegaly } & Positive & 28 & 70.0 \\
\hline & Negative & 12 & 30.0 \\
\hline \multirow[t]{2}{*}{ Fever } & Positive & 36 & 90.0 \\
\hline & Negative & 4 & 10.0 \\
\hline \multirow[t]{2}{*}{ Lymphadenopathy } & Positive & 34 & 85.0 \\
\hline & Negative & 6 & 15.0 \\
\hline \multirow{2}{*}{$\begin{array}{l}\text { Testicular } \\
\text { involvement }\end{array}$} & Positive & 2 & 5.0 \\
\hline & Negative & 22 & 55.0 \\
\hline \multirow[t]{2}{*}{ CNS involvement } & Positive & 5 & 12.5 \\
\hline & Negative & 35 & 87.5 \\
\hline
\end{tabular}

The expression of NRP-1 expression and the level of ceruloplasmin oxidase and cupper in cases and control group are illustrated in Table (2). There was highly significant increase in NRP-1 expression and the level of ceruloplasmin oxidase and copper in cases compared with control group $(\mathrm{P}<0.001)$. 
ITable (2): Comparison of neuropilin-1, ceruloplasmin-oxidase and copper in cases and control group.

\begin{tabular}{|l|c|c|c|c|c|c|c|l|l|}
\hline & \multicolumn{4}{|c|}{ Cases $(\mathbf{n}=\mathbf{4 0})$} & \multicolumn{4}{c|}{ Control $(\mathbf{n}=\mathbf{4 0})$} & \multirow{2}{*}{ P-value } \\
\cline { 2 - 11 } & Mean & SD & Median & Range & Mean & SD & Median & Range & \\
\hline nuropilin- 1 (\%) & 38.03 & 13.17 & 41.00 & $16-58$ & 4.96 & 1.21 & 5.00 & $3-7$ & $<0.001$ \\
\hline $\begin{array}{l}\text { curoplasmin } \\
\text { oxidase (mg/dL) }\end{array}$ & 0.84 & 0.18 & 0.80 & $0.61-1.3$ & 0.38 & 0.13 & 0.39 & $\begin{array}{l}0.18- \\
0.58\end{array}$ & $<0.001$ \\
\hline Cupper (mcg/dL) & 163.67 & 17.15 & 164.50 & $133-210$ & 80.57 & 30.36 & 78.00 & $36-140$ & $<0.001$ \\
\hline
\end{tabular}

Correlation between NRP-1 and laboratory parameters are illustrated in Table (3). There was no significant correlation between NRP-1 and any of the parameters.

Table (3): Correlation between NRP-1 and laboratory parameters.

\begin{tabular}{|c|c|c|}
\hline \multirow{2}{*}{} & \multicolumn{2}{|c|}{ Neuropilin-1 } \\
\cline { 2 - 3 } & r & P-value \\
\hline TLC $((\mathbf{X ~ 1 0 9 / L )})$ & 0.073 & 0.656 \\
\hline Hb(gm/dL) & 0.215 & 0.184 \\
\hline PLTs $((\mathbf{X}$ 109/L) & -0.009 & 0.955 \\
\hline PB Blasts (\%) & -0.173 & 0.287 \\
\hline BM Blasts (\%) & -0.053 & 0.746 \\
\hline
\end{tabular}

Correlation between NRP-1 and the other markers (ceruloplasmin-oxidase and copper) are illustrated in Figure (2). There was no significant correlation between NRP- 1 and ceruloplasmin oxidase or copper $(r=-0.131, P=0.422$ and $r=-$ $0.205, \mathrm{P}=0.205$ respectively).

(A) (B)
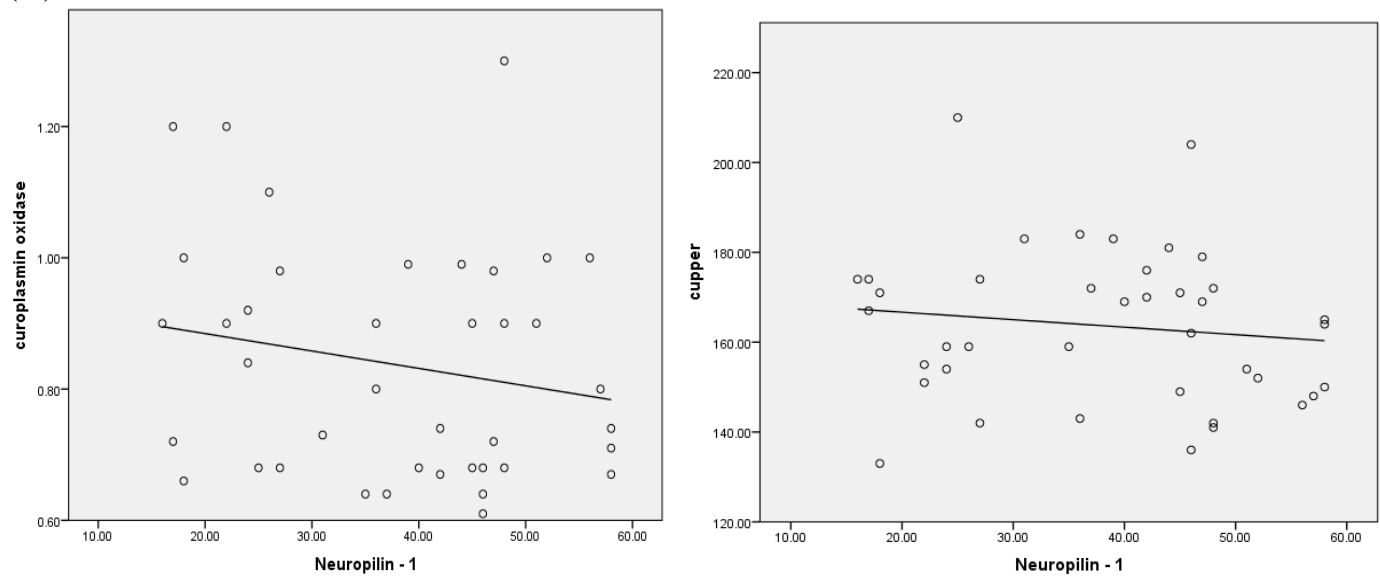

Figure (2): A: Correlation between neuropilin-1 (NRP-1) and ceruloplasmin-oxidase. B: Correlation between NRP-1 and copper.

Correlation between ceruloplasmin-oxidase and copper are illustrated in Table (4).There was an inverse correlation between ceruloplasmin oxidase and copper $(\mathrm{r}=-0.333, \mathrm{P}=0.036)$.

Table (4): Correlation between ceruloplasmin-oxidase and copper.

\begin{tabular}{|l|l|l|}
\hline \multirow{2}{*}{ Copper } & \multicolumn{2}{|l|}{ Curoplasmin oxidase } \\
\cline { 2 - 3 } & $\mathrm{r}$ & $\mathrm{P}$ \\
\hline
\end{tabular}

The output data of the receiver operation characteristic (ROC) curve for discrimination power of NRP-1 to differentiate between cases and control is shown in Table (5). The cut off value was 11.5 for NRP-1. The specificity and sensitivity of neuropilin-1 were $100 \%$ and $100 \%$ respectively. 
Table (5): The output data of the receiver operating characteristic curve for discriminative power of NRP-1 to differentiate between cases and control group.

\begin{tabular}{|c|c|c|c|c|c|c|c|}
\hline \multirow[t]{2}{*}{ 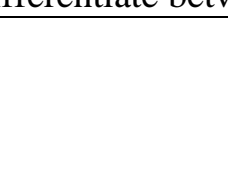 } & \multirow{2}{*}{$\begin{array}{c}\text { Area } \\
\text { Under } \\
\text { the } \\
\text { Curve }\end{array}$} & \multirow{2}{*}{$\begin{array}{c}\mathbf{P} \\
\text { value }\end{array}$} & \multicolumn{2}{|c|}{$\begin{array}{c}\text { 95\% Confidence } \\
\text { Interval }\end{array}$} & \multirow{2}{*}{$\begin{array}{c}\text { Cut } \\
\text { off } \\
\text { value }\end{array}$} & \multirow{2}{*}{ Sensitivity \% } & \multirow{2}{*}{ Specificity \% } \\
\hline & & & $\begin{array}{l}\text { Lower } \\
\text { Bound }\end{array}$ & $\begin{array}{l}\text { Upper } \\
\text { Bound }\end{array}$ & & & \\
\hline $\begin{array}{l}\text { Neuropilin- } \\
\text { 1(NRP-1) }\end{array}$ & 1.000 & $<0.001$ & 1.000 & 1.000 & 11.5 & 100 & 100 \\
\hline
\end{tabular}

This means that NRP-1 is a specific and sensitive marker for discrimination between ALL cases and control group.

\section{DISCUSSION}

Progress in the understanding and treatment of pediatric acute lymphoblastic leukemia over the past 60 years has been remarkable and it is now expected that 80 $85 \%$ of children diagnosed will be cured of this disease ${ }^{(\mathbf{1})}$.

A direct role for NRP-1 within tumor cells has also been postulated. As data continue to accumulate pointing to a role for NRP-1 in cancer, the promise for targeting this pathway is beginning to unfold ${ }^{(1)}$.

This study found that there was a highly significant statistical increase in level of NRP-1 in BALL patients compared with control group $(\mathrm{P}<0.001)$.

This is in concordance with Beesley et al. ${ }^{(9)}$ that found that NRP-1 is a marker of pediatric ALL and its expression in B-ALL patients was associated with relapse and adverse clinical outcome.

This is also in agreement with Karajalainen $\boldsymbol{e t}$ al. (10) who found a significant increase in NRP-1 expression in all patients with B-ALL and two thirds of AML cases.

While Coustan-Smith et al. (11), found that NRP-1 expression is increased in both ALL and AML patients, without significant difference between AML and ALL patients.

Also, Meyerson et al. ${ }^{(12)}$ found that NRP-1 is expressed on B-ALL blasts and stated that NRP-1 is not expressed on normal peripheral blood cells. It also found that NRP-1 was weakly and partially expressed on early and late B-cell progenitors while it was absent on mature B-cells so it is lost at later stages of B-cell maturation.

Solly et al. ${ }^{(13)}$, found that the expression of NRP1 was extremely low or undetectable on regenerative bone marrow of B-ALL patients during chemotherapy and it was one of the most differentially expressed marker between ALL and normal B-cell precursors so, it is a useful marker of minimal residual disease of B-ALL.

Younan et al. ${ }^{(14)}$, found thatNRP-1 expression was associated with acute leukemia and its level might serve as an indicator for disease severity and progression.

Hagag and Nosair ${ }^{(15)}$ stated that NRP-1 was significantly higher in B-ALL patients than control group. They also stated that NRP-1 expression was higher in patients who subsequently relapsed or later died compared to those who achieved and maintained complete remission.
Also, El Bordiny et al. ${ }^{(16)}$, found that NRP-1 was expressed in almost all B-lineage ALL patients and it might be a useful marker I MRD detection of B-lineage ALL patients.

Radwan et al. ${ }^{(17)}$, stated that NRP-1 expression was significantly higher in AML and ALL patients compared with non-hematological malignancy.

Abaza et al. ${ }^{(18)}$, stated that NRP-1 expression was significantly expressed in ALL patients compared with regenerative bone marrow specimens.

This study showed that there was no significant correlation between NRP-1 expression and the percentage of blasts in peripheral blood and bone marrow ( $\mathrm{r}=-0.173, \mathrm{P}=0.287, \mathrm{r}=-0.053, \mathrm{P}=0.746$ respectively).

This is in contrast with EL-Bordiny et al. ${ }^{(16)}$ and Radwan et al. ${ }^{(17)}$ that stated that there was a significant positive correlation with the percentage of bone marrow blasts $(\mathrm{P}=0.039, \mathrm{P}=0.02$ respectively). This difference may be due to our study shows the expression of NRP-1 on peripheral blood blasts while theirs shows the expression of the marker on bone marrow blasts.

There was no significant correlation between NRP-1 with total leukocytic count (TLC), hemoglobin $(\mathrm{Hb})$ and platelets $(\mathrm{r}=0.073, \mathrm{P}=0.656, \mathrm{r}=0.215, \mathrm{P}=0.184$ and $\mathrm{r}=-0.009, \mathrm{P}=0.955$ respectively).

This is in agreement with Radwan et al. ${ }^{(17)}$ that found that there was no significant correlation between NRP-1 with TLC, $\mathrm{Hb}$ and platelets.

Hagag and Nosair ${ }^{(15)}$ found that there was a direct correlation between NRP-1 and TLC, while there was no significant correlation with $\mathrm{Hb}$ and platelets.

This study also found that the levels of ceruloplasmin-oxidase and cupper were significantly higher in B-ALL patients compared with the control group $(\mathrm{P}<0.001)$. This is in agreement with Mehdi et al. (19), that found that there was significant increase in both ceruloplasmin oxidase and copper in ALL patients compared with healthy controls.

Gadjevi et al. (8), who found that the concentration of ceruloplasmin-oxidase was higher in patients with malignant hematological disease (including ALL) compared with controls, and remains very high after chemotherapy. They suggested that oxidative stress might lead to compensatory increased level of the ceruloplasmin in these patients due to the ability of ceruloplasmin to react with and scavenge toxic oxygen species. 
Sgarbieri et al. ${ }^{(7)}$, found that serum copper was significantly higher in children with ALL at diagnosis when compared with healthy controls, and decreased with the beginning of treatment.

\section{CONCLUSION}

It could be concluded that Neuropilin-1 can be used as a routine marker for diagnosis of ALL.

Ceruloplasmin oxidase and copper are increased in BALL patients, so further studies are recommended to detect if they have impact on the etiology of this disease.

\section{REFERENCES}

1. Bagri A, Tessier-Lavigne $M$, Watts RJ (2009): Neuropilins in tumor biology. Clin Cancer Res., 15(6):1860-4.

2. Pellet-Many C, Frankel P, Jia H et al. (2008): Neuropilins: structure, function and role in disease. Biochem J., 411(2):211-26.

3. Grandclement C, Borg C (2011): Neuropilins: a new target for cancer therapy. Cancers (Basel), 3(2):1899-928.

4. Chaudhary B, Khaled YS, Ammori BJ et al. (2014): Neuropilin 1: function and therapeutic potential in cancer. Cancer Immunol Immunother., 63(2):81-99.

5. Vales A, Kondo R, Aichberger KJ et al. (2007): Myeloid leukemias express a broad spectrum of VEGF receptors including neuropilin-1 (NRP-1) and NRP-2. Leuk Lymphoma, 48 (10):1997-2007.

6. Prud'homme GJ, Glinka Y (2012): Neuropilins are multifunctional coreceptors involved in tumor initiation, growth, metastasis and immunity. Oncotarget., 3(9):92139.

7. Sgarbieri R, Fisberg M, Tone L et al. (2006): Nutritional assessment and serum zinc and copper concentration among children with acute lymphocytic leukemia: a longitudinal study. Sao Paulo Med J., 124 (6):316-20.

8. Gadjeva V, Kutchukova D, Aladjov E et al. (2005): Correlation between plasma malondialdehyde and ceruloplasmin activity in patients with malignant haematological diseases. Trakia Journal of Sciences, 3(2): 29-33.
9. Beesley AH, Cummings AJ, Freitas JR et al. (2005): The gene expression signature of relapse in pediatric acute lymphoblastic leukemia: Implications for mechanisms of therapy failure. Br J Haematol., 131:447-56.

10. Karjalainen K, Jaalouk DE, Bueso-Ramos CE et al. (2011): Targeting neuropilin-1 in human leukemia and lymphoma. Blood, 117(3):920-27.

11. Coustan-Smith E, Song G, Clark C et al. (2011): New Markers for Minimal Residual Disease in Acute Lymphoblastic Leukemia. Blood, 117(23): 6267-6276.

12. Meyerson HJ, Blidaru G, Edinger A et al. (2012): NRP1/CD304 Expression in Acute Leukemia, A Potential Marker for Minimal Residual Disease Detection in Precursor B-Cell Acute Lymphoblastic Leukemia. American Journal of Clinical Pathology, 13:39-50.

13. Solly F, Angelot F, Garand R et al. (2012): CD304 is preferentially expressed on a subset of B - lineage acute lymphoblastic leukemia and represents a novel marker for minimal residual disease detection by flow cytometry. Cytometry, 81:17 -24.

14. Younan S, Elhoeiny S, Hammam A et al. (2012): Role of Neuropilin-1 and its Expression in Egyptian Acute Myeloid and Acute Lymphoid Leukemia Patients. Leukemia Res., 36:169-173.

15. El Bordiny M, El Ghandour A, Mansour A et al. (2016): NRP-1/CD304 expression in acute leukemia: a potential marker for minimal residual disease detection in acute leukemia. Egyptian Journal of Hematology, 41 (2): 81-86.

16. Hagag A, Nosair $\mathbf{N}$ (2015): Prognostic Impact of Neuropilin-1 Expression in Egyptian Children with Blineage Acute Lymphoblastic Leukemia. Mediterr J Hematol Infect Dis., 7(1): e2015009

17. Radwan S, Allam A, Aziz S et al. (2018): Study of Neuropilin-1/ CD304 Expression in Leukemogenesis. Journal of Hematology and Oncology Research, 3(1): 1623.

18. Abaza HM, Alfekry MA, Eissa DS et al. (2018): Neuropilin-1/CD304 Expression by Flow Cytometry in Pediatric Precursor B-Acute lymphoblastic Leukemia: A Minimal Residual Disease and Potential Prognostic Marker. J Pediatric Hematol Oncol., 40(3):200-207.

19. Mehdi W, Yousef F, Mahde A et al. (2015): Effects of acute lymphoblastic leukemia on ceruloplasmin oxidase, copper and several markers of oxidative damage, in children. Asian Pac J Cancer Prev., 16 (13): 5205-5210. 